\title{
The importance of pursuing a histological diagnosis in the HIV setting
}

\author{
${ }^{1} \mathrm{KA}$ Fernando, ${ }^{2} \mathrm{~S}$ Acharya, ${ }^{3} \mathrm{JT}$ Arumainayagam \\ ${ }^{1}$ Specialist Registrar in Genitourinary Medicine; ${ }^{2,3}$ Consultant in Genitourinary Medicine, Walsall Centre for Sexual Health, Walsall, \\ West Midlands, UK
}

ABSTRACT A 40-year-old heterosexual African man was admitted with fever, night sweats and lymphadenopathy. His human immunodeficiency virus antibody test was positive. Initial lymph node biopsy revealed reactive hyperplasia only. He was empirically treated for tuberculosis. However, symptoms persisted and a repeat lymph node biopsy was obtained, which confirmed Hodgkin's disease. This emphasises the need to pursue histological diagnosis in such patients with persisting symptoms.

KEYWORDS HIV, Hodgkin's disease, lymphadenopathy, tuberculosis
Published online December 2009

Correspondence to K Fernando, Walsall Centre for Sexual Health, Walsall Hospitals NHS Trust, Pleck Road, Walsall WS2 7BR, UK

DECLARATION OF INTERESTS No conflict of interests declared.

\section{CASE REPORT}

A 40-year-old heterosexual Zimbabwean man was admitted to hospital with a pyrexial illness. Serology on presentation included C-reactive protein of $82 \mathrm{mg} / \mathrm{l}$ and haemoglobin of $1 \mathrm{l} .3 \mathrm{~g} / \mathrm{dl}$. His total and differential white cell counts were within the normal range. A chest X-ray was normal.

As part of the investigative work-up for pyrexia of unknown origin, a computed tomography (CT) scan of the thorax, abdomen and pelvis was performed. This revealed widespread para-aortic lymphadenopathy and enlarged nodes in the left iliac fossa. Additional history was notable for weight loss and drenching night sweats over the preceding weeks.

An antibody test for human immunodeficiency virus (HIV) was positive, and HLA B570I was negative. Baseline CD4 count was 265 cells $/ \mathrm{mm}^{3}$ and HIV RNA viral load was 444,000 copies $/ \mathrm{ml}$. Highly active antiretroviral therapy (HAART) was commenced in the form of abacavir/lamivudine and efavirenz.

Working diagnoses at this stage included HIV-related reactive lymphadenopathy, mycobacterial (atypical or tuberculous) infection and neoplastic disorders.

Despite repeatedly negative investigations for tuberculosis (TB), including sputum, early morning urine samples and a gamma interferon release assay (T-spot TB), the patient was commenced on anti-tuberculous therapy as his presenting picture was consistent with TB.

A para-aortic lymph node was biopsied under CT control. Histology was consistent with reactive hyperplasia. There was no evidence of TB. Symptoms began to resolve over the subsequent few weeks and the patient tolerated anti-tuberculous therapy well.

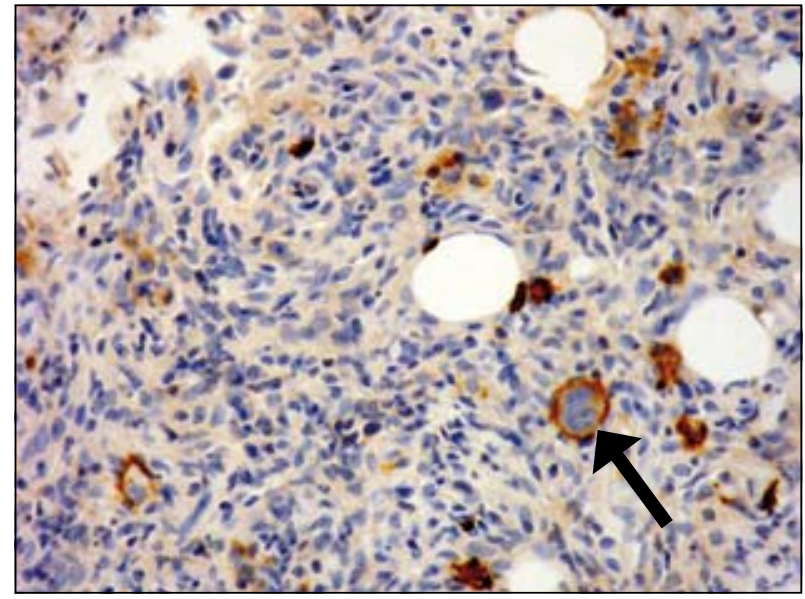

FIGURE I Histological specimen of pelvic mass. These histological features are consistent with the Reed-Sternberg malignant cell (indicated by arrow), diagnostic of classical Hodgkin's disease. The brown stain indicates CDI5 positivity.

Four months later he presented with recurrence of fever and night sweats, now in conjunction with epigastric pain and diarrhoea. He reported weight loss of $10 \mathrm{~kg}$ in the preceding six weeks. A repeat CT scan revealed further enlargement of the para-aortic lymph nodes and now a large lymph nodular mass in the left pelvis and lower abdomen.

An ultrasound-guided biopsy was obtained from this mass. Immunohistochemical staining of the biopsy revealed a number of bi- and multi-lobar cells strongly positive for Epstein-Barr virus (EBV), CDI5 and CD30. Staining for leucocyte-common antigen and human herpes virus 8 was negative. Ziehl-Neelsen stain was also negative.

These histological features are consistent with the Reed-Sternberg malignant cell (Figure I), diagnostic of classical Hodgkin's disease. A subsequent bone marrow aspirate revealed no infiltration. 
The patient completed six cycles of ABVD (doxorubicin, bleomycin, vinblastine, dacarbazine) chemotherapy and at the time of the submission of this manuscript had responded successfully. Follow-up CT imaging showed a marked reduction in the size of the pelvic mass.

\section{DISCUSSION}

The aetiology of Hodgkin's disease is unclear. In the HIV setting a high degree of EBV positivity is observed. Hodgkin's disease is a non-AIDS defining malignancy. Retrospective studies have suggested an increase in the incidence of Hodgkin's disease in the post-HAART era.'

It has been suggested that the survival and proliferation of the malignant Reed-Sternberg cells of Hodgkin's disease are enhanced by an increasing CD4 count. This may occur by means of mutually beneficial cytokine interaction between the Reed-Sternberg cell and CD4 lymphocyte, preventing apoptotic mechanisms.'

In the HIV-negative setting, the most commonly seen subtype of Hodgkin's disease is nodular sclerosing. In HIV-positive individuals, however, mixed cellularity and lymphocyte-depleted Hodgkin's lymphoma are the most common subtypes, which, unfortunately, confer a worse prognosis.

The recently published British HIV Association (BHIVA) guidelines on the management of HIV-associated malignancy recommend the use of HAART in all patients with HIV-associated Hodgkin's disease. ${ }^{2}$ Improved overall survival and disease-free survival in such patients has been observed in the post-HAART era. ${ }^{3.4}$

Inhibition of both the cytochrome P450 enzyme and glycoprotein efflux protein systems has been observed with the use of boosted protease-inhibitor HAART regimens. ${ }^{2}$ This can potentially lead to increased chemotherapy toxicity. Therefore, non-nucleoside reverse transcriptase inhibitor-based HAART regimes are preferred for individuals with HIV-associated malignancies.

As first-line treatment, the BHIVA recommends the chemotherapy regime of ABVD for HIV-associated Hodgkin's disease. ${ }^{2}$

Consideration of prophylaxis against opportunistic infection $(\mathrm{Ol})$ is important. ${ }^{4}$ Chemotherapy agents can cause further immunosuppression in the HIV-infected individual. Despite a CD4 count of greater than 200 cells $/ \mathrm{mm}^{3}$, OI prophylaxis should be considered if the patient is to receive chemotherapy regimes where the CD4 count is predicted to decline below the threshold for initiation of Ol prophylaxis. ${ }^{2}$

\section{CONCLUSION}

Our case report firstly highlights the importance of pursuing alternative diagnoses when faced with persisting symptoms refractory to initial treatment, and secondly increases awareness of the possible rise in incidence of Hodgkin's disease with improved immunity in the postHAART era.

\section{REFERENCES}

I Biggar RJ, Jaffe ES, Goedert JJ et al. Hodgkin lymphoma and immunodeficiency in persons with HIV/AIDS. Blood 2006; 108:3786-91. doi: 10.1 182/blood-2006-05-024109

2 Bower M, Collins S, Cottrill C et al. British HIV Association guidelines for HIV-associated malignancies 2008. HIV Med 2008; 9:336-88. doi:I0.IIII/j. I 468-1293.2008.00608.x

3 Hoffman $\mathrm{C}$, Chow $\mathrm{KU}$, Wolf $\mathrm{E}$ et al. Strong impact of highly active antiretroviral therapy on survival in patients with human immunodeficiency virus-associated Hodgkin's disease. $\mathrm{Br} J \mathrm{Haematol}$ 2004; I25:455-62. doi: I0.III I/j. I365-2| |4I.2004.04934.X 